\title{
Probióticos, terceira idade e Saúde Pública
}

\author{
Probiotics, third age and Public Health
}

\author{
Sueli Essado Pereira' \\ Kelry Alves Ramos ${ }^{1}$ \\ ${ }^{1}$ Pontifícia Universidade Católica de Goiás. Goiânia/GO, Brasil.
}

A Organização Mundial da Saúde (OMS) ${ }^{1}$ define idoso a partir da idade cronológica, portanto idosa é aquela pessoa com 60 anos ou mais, em países em desenvolvimento, e com 65 anos ou mais em países desenvolvidos. A idade cronológica não é um marcador preciso para as mudanças que surgem no envelhecimento, pois existem diferenças significativas de acordo com o estado de saúde, participação e níveis de independência entre pessoas que possuem a mesma idade ${ }^{2}$. No entanto, o desejo de longevidade vem acompanhado da necessidade de manter estado de saúde e qualidade de vida ${ }^{3}$.

De acordo com dados estatísticos, em 2002 a população brasileira de idosos cresceu 500\% desde 1965 (processo de 40 anos), com estimativa de chegar a 32 milhões em 2020, números surpreendentes se comparados com os de outros países, sendo resultado de uma política crescente com relação aos serviços de saúde 4 . Por outro lado, mesmo que os idosos consigam reduzir a prevalência de doenças crônicas, ainda permanece o desafio com relação à redução da capacidade funcional e fisiológica ${ }^{3}$. Devido a essas mudanças fisiológicas, bioquímicas, psicológicas e funcionais que acometem o processo de envelhecimento, permitem que o idoso seja mais susceptível a doenças, principalmente as de caráter crônicodegenerativas, afetando diretamente o estado nutricional dessa população ${ }^{5-7}$. Alterações no funcionamento digestivo (ingestão, digestão, absorção, utilização dos nutrientes), diminuição do metabolismo basal, redistribuição da massa corporal, mudanças na percepção sensorial, comprometem ainda mais o estado de saúde e as necessidades nutricionais dos idosos ${ }^{8-9}$.

Ainda considerando essas alterações fisiológicas na população idosa, associa-se a elas um declínio da função imunológica, podendo ocasionar baixa resposta às vacinas e predisposição às doenças infecciosas e não infecciosas. Sendo assim, nessa fase ocorrem mudanças orgânicas e teciduais, e, além disso, a microbiota intestinal difere da dos adultos, devido a um crescente número de bactérias prejudiciais, tais como: Clostridium perfringens e enterobactérias, em comparação com a microbiota essencial, como dos grupos lactobacilos e bifidobactérias ${ }^{10}$. Estudos atuais já alertam para esse desequilíbrio na microbiota, denominado de disbiose ${ }^{11}$, podendo promover tanto constipação como diarreia, cujos índices são alarmantes nesse público ${ }^{12}$. Os sinais e sintomas da disbiose podem aparecer por causas primárias na terceira idade, como situações contínuas de má alimentação, estresse, má digestão, irregularidades no trânsito intestinal, pH intestinal e estomacal alterado, hipocloridria, além de reduzido estado imunológico. Como fatores secundários estão associados uso indiscriminado de medicamentos controlados como antibióticos, anti-inflamatórios hormonais e não 
hormonais, laxantes, assim como exposição excessiva aos poluentes ambientais e alimentares, doenças como câncer e síndrome de imunodeficiência, além de outras síndromes que acometem o sistema gastrointestinal e consequentemente agridem a estabilidade do estado nutricional ${ }^{13}$.

Todas essas evidências demonstram a necessidade de promover na Saúde Pública um olhar mais diferenciado e resolutivo à terceira idade, promovendo uma saúde preventiva mediante o consumo de alimentos funcionais, com reeducação alimentar e nutricional envolvendo uso de alimentos fontes de probióticos e prebióticos ${ }^{14}$. Os alimentos funcionais foram assim designados pelos japoneses, considerando princípios medicinais naturais desde a época de Hipócrates (500 a.C.), quando alegava que nosso alimento deveria ser nosso remédio e nosso remédio o alimento. Houve um olhar diferenciado para certos alimentos com propriedades preventivas e curativas, cuja prática de consumo demonstrava diversos benefícios, reduzindo prevalência de doenças crônicas e consequente diminuição nos gastos públicos. Os alimentos funcionais mais indicados para saúde do idoso e com maior número de pesquisas são os fornecedores de fibras alimentares e/ou prebióticos (como aveia e sementes de linhaça), assim como fonte de carotenoides (tomate), fonte de ômega 3 (óleo de canola e linhaça, e peixes), fonte de selênio e vitamina E (castanhas e amêndoas) ${ }^{15}$, além de outros alimentos essenciais e fontes de probióticos (derivados lácteos como coalhada, iogurte, leite fermentado) entre outros também com valores nutricionais ${ }^{10,12}$.

Os estudos nessa área ainda são insuficientes e controversos, mas sabe-se que dos levantamentos realizados, uma alimentação balanceada para esse público pode sim prevenir diversos sinais e sintomas que promovem as doenças crônicas não degenerativas. Alguns levantamentos científicos demonstraram ainda os benefícios preventivos com a suplementação de cepas probióticos dos gêneros lactobacilos e bifidobactérias, ressaltando uma grande relevância social - a indicação de mais estudos com esse tema - para definir com mais especificidade o panorama da saúde pública senil e respectivas terapias alternativas.

\section{REFERÊNCIAS |}

1. Organização Mundial da Saúde. Global burden of disease. Genebra: OMS; 2002.

2. Organização Pan-Americana da Saúde. Envelhecimento ativo: uma política de saúde. Brasília: OPAS; 2005.

3. Veras RP, Oliveira MO. Envelhecer no Brasil: a construção de um modelo de cuidado. Ciênc Saúde Coletiva. 2018; 23(6):1929-36.

4. Closs E, Schwnake CHA. A evolução do índice de envelhecimento no Brasil, nas suas regiões e unidades federativas no período de 1970 a 2010. Rev Bras Geriatr Gerontol. 2012; 15(3):443-58.

5. Nascimento CM, Ribeiro AQ, Sant'Ana LFR, Oliveira RMS, Franceschini SCC, Priore SE. Estado nutricional e condições de saúde da população idosa brasileira: revisão da literatura. Rev Med Minas Gerais. 2011; 21(2):174-80.

6. Tirapegui J, Ribeiro LSM. Avaliação nutricional: teoria e prática. São Paulo: Guanabara Koogan; 2011.

7. Paz RC, Fazzio DMG, Santos ALB. Avaliação nutricional em idosos institucionalizados. Revisa. 2012; 1(1):9-18.

8. Malta MB, Papini SJ, Corrente JE. Avaliação da alimentação de idosos do município paulista: aplicação do Índice de Alimentação Saudável. Ciênc Saúde Coletiva. 2013; 18(2):377-84.

9. Martins MV, Souza JD, Franco FS, Martinho KO, Tinôco ALA. Consumo alimentar de idosos e sua associação com o estado nutricional. HU Revista. 2016; 42(2):125-31.

10. Vulevic J, Drakoularakou A, Yaqoob P, Tzortzis G, Gibson GR. Modulation of the fecal microflora profile and immune function by a novel trans-galactooligosaccharide mixture (B-GOS) in healthy elderly volunteers. Am J Clin Nutr. 2008; 88(5):1438-46. 
11. Chan YK, Estaki M, Gibson DL. Clinical consequences of diet-induced dysbiosis. Ann Nutr Metab. 2013 ; 63 Supl. 2:28-40.

12. Pereira LS, Oliveira LR, Santos MT, Barbi T, Calil AM. Benefícios da utilização de prebióticos, probióticos e simbióticos em adultos e idosos. Rev Geriatr Gerontol. 2014; 8(1):77-81.

13. Conrado BA, Souza SA, Mallet ACT, Souza EB, Neves AS, Saron MLG. Disbiose intestinal em idosos e aplicabilidade dos probióticos e probióticos. Cadernos UniFOA. 2018; 13(36):71-8.

14. Torres JDRV, Nobre SAM, Silva JR, Caldeira MTG, Silva TN, Torres SAS, et al. Microbiota intestinal e associações com desordens clínicas em função da faixa etária de idosos: um estudo analítico transversal. Estud Interdiscipl Envelhec. 2016; $21(1): 263-81$.

15. Silva IMC, Sá EQC. Alimentos funcionais: um enfoque gerontológico. Rev Bras Clin Med. 2012; 10(1):24-8. 